\title{
LAS CRISIS DE GOBERNABILIDAD DE BOLIVIA (2000-2005), SU RELACIÓN CON LA DEBILIDAD DEL ESTADO Y LA SEGURIDAD REGIONAL ${ }^{*}$
}

\author{
CAROLINA SAMPÓ**
}

\begin{abstract}
RESUMEN
Durante la última década ha quedado de manifiesto cómo la seguridad de los Estados depende en gran parte de la capacidad que estos tengan de resolver y contener sus problemas internos. Es por eso que, más allá de los años de democracia que la región muestra con orgullo y de la desactivación de casi todas las hipótesis de conflicto interestatales, en Latinoamérica se hace necesario prestar atención en cómo la debilidad de los Estados posibilita el avance de las amenazas no convencionales, particularmente del crimen organizado en sus distintas variaciones (especialmente narcotráfico y contrabando).

En este sentido, las características de los Estados de la región permiten en gran medida el asentamiento y la reproducción de organizaciones criminales de carácter transnacional. La propia debilidad del Estado, así como el alcance de la cuestión indígena y de la cultura política, particularmente en la Región Andina (compuesta por Colombia, Venezuela, Ecuador, Perú y Bolivia) ponen de manifiesto las carencias del modelo de Estado existente y abren la puerta al ascenso de nuevos actores. En este contexto, el presente trabajo procura mostrar cómo, en el caso de Bolivia (tomado como caso testigo), las crisis de gobernabilidad de los años 2003 y 2005 profundizaron la inseguridad regional generada desde ese territorio.
\end{abstract}

Palabras clave: Seguridad regional, crisis de gobernabilidad, Bolivia, debilidad institucional, narcotráfico.

* Este paper fue escrito en el marco de mi tesis doctoral publicada bajo el título "Crisis de gobernabilidad, desafíos a la seguridad regional: Los casos de Bolivia y Ecuador (2000-2005)”, Editorial Académica Española, Alemania, 2012.

** Doctora en Ciencias Sociales (UBA). Becaria posdoctoral del Conicet. Magíster en Estudios Internacionales (UTDT). Licenciada en Ciencia Política (UBA). Docente de la UBA. Argentina. carosampo@gmail.com

$\infty \quad$ Fecha de recepción: 031212

Fecha de aceptación: 030513 


\title{
GOVERNABILITY CRISIS IN BOLIVIA (2000 - 2005) ITS RELATIONSHIP WITH STATE WEAKNESS AND REGIONAL SECURITY
}

\begin{abstract}
During the last decade, there is enough evidence to demonstrate that the state' security depends mostly in their ability to solve and contain its internal problems.

For that reason, more than the years of democracy the region proudly shows, and the defusing of most of the interstate conflict hypothesis, in Latin America it is necessary to focus in the fact that how the weakness of the states, favors the advance of no conventional threats, particularly, organized crime in its various faces (specially drug trafficking y smuggling). In that sense, the characteristics of the states of the region, allow in a certain way, the establishment and reproduction of transnational crime organizations. The state's weakness, as well as the indigenous problem and political culture, particularly in the Andean Region (Colombia, Venezuela, Peru and Bolivia) demonstrate the shortness of the current state's model and open the path to the presence of new actors. In this given context, this article tries to show how in the Bolivian case (as a witness) the governability crisis of 2003 and 2005, ended in a more profound regional insecurity generated from this country.
\end{abstract}

Key words: Regional security, governability crisis, Bolivia, institutional weakness, drug - trafficking.

\section{Introducción a la problemática}

Desde las respectivas aperturas democráticas los países de la región sudamericana mostraron diversas dificultades para consolidar el régimen político y, especialmente, fortalecer sus instituciones. Como consecuencia, la gobernabilidad y, principalmente sus deficiencias, adquirieron un rol fundamental en el devenir de nuestras democracias; especialmente en la Región Andina (compuesta por Colombia, Ecuador, Bolivia, Perú y Venezuela). Entendemos gobernabilidad como la capacidad del Estado de responder a las demandas generadas desde la sociedad civil, así como de articular los diferentes intereses sociales mediando sus diferencias. 
La idea de gobernabilidad se vincula con la capacidad de los gobernantes, ciudadanos e intermediarios de lograr consensos que hagan posible formular políticas que permitan responder equilibradamente aquello que la sociedad espera del gobierno ${ }^{1}$. En este marco, una crisis refleja la existencia de un desequilibro permanente entre las demandas de la sociedad civil y las respuestas del gobierno, mientras que se deja de manifiesto la incapacidad para articular intereses diversos.

Existe cierto consenso sobre las áreas a tener en cuenta a la hora de evaluar la situación de cada régimen, de acuerdo con Camou², estas son: la capacidad que tiene el gobierno de mantener o al menos restaurar rápidamente un nivel mínimo de orden en el marco de la ley; la capacidad del gobierno para desarrollar una gestión económica eficaz; la capacidad del gobierno de garantizar servicios sociales mínimos y promover el bienestar social; y la capacidad del sistema político para incorporar, restringir o acomodar a grupos o individuos que quieran influir en el juego político con el propósito de permitir el flujo eficaz en la toma de decisiones.

En nuestra opinión, existen dos fuentes de problemas de gobernabilidad: por un lado, aquellos generados desde la sociedad civil, que se moviliza y lleva adelante determinadas demandas hacia el sistema político; y, por el otro, los problemas generados a partir de la falta de articulación de intereses de los dirigentes políticos, que optan por confrontar en lugar de cooperar en ámbitos como el Poder Legislativo, poniendo en jaque el statu quo vigente.

Aunque las crisis de gobernabilidad suelen combinar ambas fuentes, parece ser la presión generada desde la sociedad civil la que puede provocar la ruptura institucional. Sin embargo, no hay que desestimar el papel de los representantes ya que, desde el momento en que la presión ejercida por las protestas y movilizaciones sociales provoca una fractura en la clase política, es difícil que se generen respuestas positivas a las demandas de la sociedad civil. Lejos de eclosionar de un día para el otro, las manifestaciones ciudadanas expresan un proceso de deterioro progresivo cuyas representaciones se plasman en distintos ámbitos de la vida cotidiana.

En este contexto, queda claro que la estabilidad de los gobiernos depende en gran medida de la eficacia y eficiencia que demuestren a la hora de dar respuestas a las demandas de la sociedad civil. De lo contrario, y como consecuencia de la deslegitimación que comienza por los partidos políticos en gene-

TOMASSINI, Luciano. Gobernabilidad y políticas públicas en América Latina. En Fernando Carrillo Flórez (ed.), Democracia en déficit. Gobernabilidad y desarrollo en América Latina, Washington DC, BID, 2001.

2 CAMOU, Antonio. Gobernabilidad y democracia, Cuadernos de divulgación de la cultura democrática Nro. 6, México Instituto Federal Electoral, 2001. 
ral y los gobernantes en particular, es posible que el gobierno deba entregar el mando antes de tiempo 3 .

En el marco descripto, Bolivia aparece como un caso crítico que además pone de manifiesto la relación establecida entre las crisis de gobernabilidad y los problemas de seguridad de la regional. El tráfico de drogas y el contrabando aparecen como las cuestiones más candentes a nivel local y regional. Tal como veremos más adelante, tanto en el año 2003 como en el 2005, Bolivia vivió momentos de ruptura institucional que comenzaron como virulentas crisis de gobernabilidad y culminaron con la renuncia del Presidente en ejercicio. Durante esos momentos de crisis, las actividades ilícitas pudieron desarrollarse con "mayor facilidad" en territorio boliviano ya que las preocupaciones centrales de la clase política estaban focalizadas en otro lado; mientras que para algunos sectores de la sociedad civil estas actividades reportaban ganancias e incluso proporcionaban una solución a algunas de las demandas sociales que el gobierno no había podido resolver.

\section{La vuelta a la democracia en Bolivia}

El colapso del Estado Burocrático Autoritario 4 a partir del cual se produjo el regreso a la democracia en Bolivia, hizo que los actores políticos tuvieran que aprender a participar en el juego democrático sin preparación de ningún tipo. Como consecuencia, algunas de las reglas de juego -especialmente las informales- fueron definidas durante el juego democrático.

La consolidación del régimen en Bolivia fue un proceso largo que estuvo fuertemente vinculado al desarrollo de la denominada Democracia Pactada. De acuerdo con Vargas y Córdova ${ }^{5}$, esta consistía en una dinámica de coaliciones parlamentarias que además de garantizar un cierto grado de gobernabilidad pro-

3 En nuestra región abundan ejemplos de gobiernos que debieron entregar el mando antes de tiempo. Algunos de ellos fueron: Raúl Alfonsín y Fernando de la Rúa, en Argentina; Raúl Cubas Grau y Fernando Lugo, en Paraguay; Fernando Collor de Melo, en Brasil; y Carlos Andrés Pérez, en Venezuela.

4 El BA es un tipo particular de Estado, resultante de la especificidad histórica de cada caso, cuyas características principales son: 1) su principal base social es la gran burguesía; 2) sus actores se imponen dos grandes tareas: reimplantar el orden a partir de la resubordinación del sector popular y "normalizar" la economía; 3) excluye políticamente a un sector popular previamente activado; 4) la mencionada exclusión conlleva la supresión de la ciudadanía, de lo popular y de la democracia política; 5) es un sistema de exclusión económica del sector popular a favor de la gran burguesía; 6) promueve una mayor transnacionalidad; 7) implica el "encogimiento" de la Nación porque rompe con el discurso homogeneizante; 8) busca despolitizar cuestiones sociales; 9) su régimen implica el cierre a los canales democráticos de acceso al gobierno y a la representación, esos lugares quedan reservados para las altas cúpulas de las FF.AA. y de grandes empresas. Ver O’DONNELL, Guillermo. El Estado Burocrático Autoritario. Triunfos, derrotas y crisis. Bs. As. Editorial de Belgrano, 1996.

5 VARGAS R., Humberto y CÓRDOVA E., Eduardo. Bolivia: un país de re-configuraciones por una cultura de pactos políticos y de conflictos. En Seoane, José. Movimientos sociales y conflictos en América Latina, Buenos Aires, CLACSO, 2003. 
veían estabilidad al gobierno de turno respaldando sus acciones y propuestas. El sistema se adoptó luego de las dificultades enfrentadas por los primeros gobiernos democráticos, principalmente con el objetivo de alcanzar la mayoría absoluta necesaria para elegir al Presidente de la República ${ }^{6}$. Como consecuencia, los tres partidos con mayor caudal electoral llegaron a un acuerdo que dio origen al denominado presidencialismo parlamentizado.

Sin duda la gran fragmentación del sistema de partidos y la carencia de una fuerte distancia ideológica contribuyeron para que este fuera el escenario. Tan extremo era el escenario, que entre 1985 y 2002 solo un partido logró superar los 30 puntos porcentuales, al tiempo que la diferencia más importante entre un partido y otro en elecciones nacionales fue de $5 \%$ en el año $1997^{7}$. En realidad, en este contexto, era inevitable un acuerdo interpartidario entre los líderes políticos si se pretendía mantener el régimen democrático, impulsar la alternancia en el poder y obtener cierta gobernabilidad.

La Democracia Pactada consistió en la institucionalización de una democracia formal, es decir, de un régimen que quedó reducido a un marco de reglas y procedimientos formales pero que pudo mantenerse en el tiempo ${ }^{8}$. Pero para el año 2002 la coalición ya se encontraba debilitada, aunque consiguió posicionar a Sánchez de Lozada como nuevo Presidente de la República. Esta vez, la oposición liderada por Evo Morales quedó solo a dos puntos porcentuales de distancia, al tiempo que crecía la importancia del movimiento liderado por Felipe Quispe, dando lugar a la aparición del debate político-ideológico que había estado ausente durante las etapas de transición y consolidación democrática. Con una sociedad tan fragmentada y polarizada ideológicamente, la crisis venidera era fácil de prever.

\section{De la estabilidad a las crisis de gobernabilidad}

Aunque el proceso de consolidación democrática encontró la forma de "estabilizar" tanto al sistema político como a los actores sociopolíticos que lo conformaban con la Democracia Pactada, este mecanismo informal no institucionalizado simplemente dejó de funcionar.

6 HERRERO, Francisco. Sistema de Partidos y desarrollo: El caso de Bolivia. En Guerra-García, Gustavo y Sample, Kristen (coeditores). La política y la pobreza en los países andinos. Lima, International Institute for Democracy and Electoral Assistance y Asociación Civil Transparencia, 2007.

7 GARCÍA Montero, Mercedes. Bolivia. En: Alcántara, Manuel y Freidenberg, Flavia (coord.), Partidos Políticos de América Latina. Países Andinos. México D.F., Fondo de Cultura Económica e Instituto Federal Electoral, 2003, pp. 33-145.

8 ALENDA, Stéphanie. Bolivia: La erosión del pacto democrático. Quito, Ecuador Debate, agosto de 2004, pp. $119-136$ 
El pacto político no pudo evitar las severas crisis de gobernabilidad que debió sobrellevar Bolivia en los años 2003 y 2005. En mi opinión, esto se debe a la drástica modificación del sistema de partidos, pero especialmente a la presión ejercida por los movimientos sociales que pugnaban por ser movilizados e incluidos políticamente, no solo desde lo discursivo. Estos movimientos, que en un primer momento tuvieron arraigue sindical, ampliaron su base e incorporaron una dialéctica reflexiva relacionada a la reconstrucción de la identidad indígena boliviana.

Es importante destacar la erosión de la legitimidad que sufrieron los partidos políticos considerados tradicionales, ya que la misma terminó por repercutir en el cuestionamiento de la democracia tal como estaba establecida. Algo similar pasa con las desigualdades, sean sociales, económicas o étnicas, porque ponen en duda uno de los principios básicos de la democracia: la igualdad.

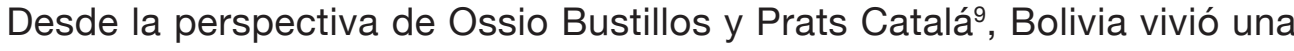
crisis del modelo de gobernabilidad resultante de la primera democratización. A pesar de sus falencias, la resolución de una crisis de gobernabilidad por vía institucional que además incorpora nuevas herramientas para manejar las tensiones sociopolíticas del régimen democrático vigente, implica sin duda un avance hacia la consolidación democrática. Como apunta Rojas Ortuste, estos quiebres pueden ser considerados una "crisis de crecimiento democrático", sin subestimar lo violento de las situaciones en las que se desarrollaron las sucesiones presidenciales de los años 2003 y $2005^{10}$.

\subsection{La primera crisis de gobernabilidad (2003)}

El escenario que tuvo que administrar el presidente Sánchez de Lozada cuando llegó al gobierno fue crítico. Principalmente se encontró con el empantanamiento de las negociaciones en torno a la erradicación de los cultivos de coca $y$, como consecuencia, con la existencia de un conflicto abierto con los sectores cocaleros, que contribuyeron a la deslegitimación de su figura frente a gran parte de la sociedad civil. De hecho, el mandatario era visto como miembro de la "oligarquía", ajeno a la realidad de la mayor parte de la población, campesinos e indígenas. Adicionalmente, el déficit fiscal superaba el $8 \%$ del Producto Bruto Interno (PBI) y los préstamos extranjeros no alcanzaban para saldar las cuentas.

9 OSSIO Bustillos, Teresa y PRATS Catala, Joan. Crisis de gobernabilidad como oportunidad democrática: el caso de Bolivia. En Congreso Internacional del CLAD sobre la Reforma del Estado y de la Administración Pública (IX Madrid, España, 2-5 Nov. 2004).

10 ROJAS Ortuste, Gonzalo. Bolivia ante el cambio: ¿Reforma pactada o revolucionarismoetnicista? Bolivia, Revista Umbrales Nro. 19: Bolivia y el contexto político actual, 2009. pp. 47-78. 
En este sentido, es necesario destacar el primer aspecto de lo que terminaría siendo una crisis de gobernabilidad severa, con posterior ruptura institucional: la polarización entre sectores sociales considerados antagónicos, como los indígenas, liderados por Morales y Quispe, y la oligarquía, encarnada por la clase política tradicional, corporizada en Sánchez de Lozada. El creciente enfrentamiento entre estos sectores fue la semilla de la destrucción del mecanismo establecido en la transición democrática con el fin de facilitar la gobernabilidad. Sin embargo, Sánchez de Lozada no parece haber tomado debida nota de la crítica situación que atravesaba la sociedad boliviana y se inclinó por intentar resolver el aspecto económico de la crisis.

El Presidente, en lugar de acercar posiciones, llevó adelante un "impuestazo"11 que motivó una nueva serie de protestas sociales de suma importancia, que culminaron con sangrientos disturbios ${ }^{12}$. En este sentido, las manifestaciones contra el gobierno comenzaron con la movilización de los jóvenes de la Universidad Popular El Alto, pero fueron ampliando su base hasta incluir a la Policía Nacional, que se negó a patrullar las calles con el fin de detener la revuelta. Los manifestantes no solo rechazaban el impuestazo, sino que exigían un aumento salarial del $40 \%{ }^{13}$. Como consecuencia, Sánchez de Lozada apeló al Ejército para que restableciera el orden público. Durante estas jornadas la institución castrense reprimió a civiles y policías sin distinción, dando lugar al denominado Febrero Negro.

Desafortunadamente, el Febrero Negro, como se conocen los disturbios del 12 y 13 de ese mes del año 2003, dejó un saldo de 33 muertos y 180 heridos, y puso de manifiesto "los rasgos estructurales de la crisis política, económica y social de Bolivia y configuraron una nueva coyuntura política de crisis, caracterizada por una degradación y una inestabilidad que colocan en riesgo el mismo proceso democrático"14.

En ese escenario, la oposición forzó al gobierno, que debió dar respuestas a los sectores más combativos, a derogar el impuestazo y a declarar la moratoria a la erradicación de cultivos de coca, al tiempo que buscaban enjuiciar al

11 El impuestazo gravaba con el $12,5 \%$ los ingresos que estuvieran por encima de cinco salarios mínimos. Con él, el gobierno pretendía recaudar los ingresos que no generaba con el Impuesto al Valor Agregado (IVA).

12 COTLER, Julio. Bolivia-Ecuador-Perú, 2003-2004: ¿Tempestad en los Andes? Real Instituto El Cano de Estudios Internacionales y Estratégicos, Área: América Latina - DT N 51/2005 (Fecha de Consulta: 08/05/11) Disponible en www.realinstitutoelcano.org/documentos/233/233_Cotler.pdf

13 WITKER Barra, Iván. Bolivia 2003 en la prensa chilena. Percepciones de la crisis en la prensa chilena y su impacto en la seguridad subregional y relaciones bilaterales. Santiago de Chile, Colección de Investigaciones ANEPE, N 11, 2005.

14 CALDERÓN, Fernando y GAMARRA, Eduardo. Crisis, inflexión y reforma del sistema de partidos en Bolivia, Revista Colombia Internacional Nro. 58, 2003, p. 13 (Fecha de ingreso: 15/09/10) Disponible en http:// colombiainternacional.uniandes.edu.co/view.php/421/1.php 
Presidente por su responsabilidad en las muertes resultantes de la represión que había ordenado.

Sánchez de Lozada entendió la precaria situación política en la que se encontraba, maniobró en consecuencia y logró superar la crisis de febrero. Sin embargo, la debilidad del gobierno había quedado expuesta y las turbulencias seguirían. Los choques entre los cocaleros y los militares en Chapare continuaron, mientras los campesinos del Altiplano siguieron cortando las carreteras con el fin de que el gobierno escuchara sus reclamos. En el otro extremo, en la Media luna boliviana ${ }^{15}$, organizaciones empresariales y comités cívicos de los departamentos orientales dejaron en claro su frustración por la paralización de la producción de gas en la zona, así como por las invasiones a tierras privadas, acusando al gobierno de ser incapaz de garantizar la propiedad privada dentro del territorio nacional ${ }^{16}$.

La gestión del Presidente estaba acorralada desde dos sectores distintos: por un lado, debido a la oposición parlamentaria pero, principalmente, por las protestas sociales, cada vez más fuertes y numerosas. Con el fin de terminar con los bloqueos y mejorar la gobernabilidad, el Presidente llamó a un diálogo nacional entre sus representantes y distintas organizaciones sociales que fracasó abruptamente a causa de la intransigencia de la oposición.

La crisis política se profundizó a partir del 15 de septiembre de 2003, cuando el gobierno lanzó una campaña informativa sobre un plan de explotación de gas natural que había diseñado. Dicho plan incluía la explotación de los yacimientos ubicados en el departamento de Tarija, por parte de un consorcio compuesto por Repsol YPF, British Gas y Panamerican Energy y contemplaba el uso de un puerto chileno para su exportación a México y Estados Unidos. Ese proyecto provocó manifestaciones en contra del plan, que se hicieron cada vez más masivas, generando una campaña antiexportadora sin precedentes. A este reclamo se sumaron las críticas al modelo neoliberal, particularmente en lo referente a las privatizaciones ${ }^{17}$.

Estos episodios se conocieron como La Guerra del Gas, que fue resultado de la profundización y polarización del conflicto y tuvo su punto más álgido en octubre de 2003. En ese momento los movimientos sociales, liderados por Morales y Quispe, decidieron cercar la ciudad de La Paz, impidiendo el ingreso de productos agrícolas y cerrando el suministro de gas. La respuesta del gobierno frente a las crecientes movilizaciones (del 12 al 14 de octubre) fue nuevamente la

\footnotetext{
Se denomina Media luna a los departamentos de Tarija, Pando, Beni y Santa Cruz de la Sierra.

COTLER, J. Loc. cit.

WITKER Barra, Iván. Loc. cit.
} 
represión, en especial en el Altiplano paceño, la ciudad de El Alto y La Paz, lo que dejó 75 muertos y más de 300 heridos, y precipitó los acontecimientos políticos ${ }^{18}$.

Las protestas Ilegaron a su clímax cuando la población de El Alto marchó sobre La Paz, exigiendo que Sánchez de Lozada abandonara sus funciones y fuera enjuiciado. A esa movilización se sumaron también los partidos políticos de oposición y la clase media, generando una presión difícil de tolerar desde el gobierno ${ }^{19}$.

La sensación de vacío de poder y de falta de gobernabilidad llegaron a su punto más álgido. El enfrentamiento volvió a generar decenas de muertos y centenares de heridos como resultado de la represión, lo que incrementó el repudio general al gobierno y terminó por producir el aislamiento y desprestigio de los partidos oficialistas. En este marco, el vicepresidente Carlos Mesa optó por distanciarse de Sánchez de Lozada, al que los militares también le retiraron su apoyo, condenándolo al abandono del poder y posterior exilio el 17 de octubre de $2003^{20}$.

Como destacan Calderón y Gamarra ${ }^{21}$, se pueden mencionar al menos cuatro elementos que llevaron a la crisis de gobernabilidad y posterior ruptura institucional de octubre de 2003; a saber: a) la explosión de conflictos sociales en todo el país, que resaltaban demandas particulares; b) la debilidad del gobierno para procesar dichas demandas; c) la articulación de esos conflictos en torno al rechazo a la explotación del gas y el reclamo de una Asamblea Constituyente ${ }^{22}$, y d) el fracaso de la mediación de la Iglesia que terminó por polarizar más el escenario sociopolítico.

En términos fácticos, la caída de Sánchez de Lozada se debió a una sucesión de situaciones más que a un episodio concreto. En primer lugar hay que destacar el agotamiento del Presidencialismo Parlamentizado como garante de la gobernabilidad, en conjunto con el incremento de la popularidad de movimientos sociales inclusivos que defendían la identidad de la Bolivia indígena. En segundo lugar, se hace necesario tener en cuenta la difícil situación económica que enfrentaba el país, de manera tal que era difícil para el Ejecutivo dar res-

18 INCHUTA Nina, Carlos E. La relación de las instituciones políticas, la forma multitud y los movimientos sociales en Bolivia. Tinkazos, vol. 11 Nro. 25, La Paz, noviembre 2008 (Fecha de ingreso: 15/08/10) Disponible en http://www.scielo.org.bo/scielo.php?script=sci_arttext\&pid=S1990-74512008000200008\&lng=es\&nrm=is s\&tlng=es

19 SUÁREZ, Hugo. Seis claves para interpretar la crisis boliviana. Memoria № 198, México, agosto de 2005 (Fecha de Ingreso 20/03/11) Disponible en http://catedras.fsoc.uba.ar/toer/articulos/txt-suarez.htm

20 COTLER, J. Loc. cit.

21 CALDERÓN, Fernando y GAMARRA, Eduardo. Op. cit. pp. 90 a 123 (Fecha de ingreso: 15/09/10) Disponible en http://colombiainternacional.uniandes.edu.co/view.php/421/1.php

22 Esta Asamblea, que se llevó adelante en el 2005, tuvo como objetivo modificar la representación política y la organización estatal para permitir la inclusión de asociaciones civiles y organizaciones indígenas en la arena política. 
puestas satisfactorias a las demandas sociales. En particular, hay que considerar la tendencia neoliberal de Sánchez de Lozada y su cercanía ideológica y cultural con Estados Unidos, que generaba cierto resquemor en los estratos más bajos de la sociedad. En tercer lugar, se deben mencionar los episodios de represión que dieron como resultado decenas de muertos y centenares de heridos, y que ampliaron la brecha entre el Presidente y la sociedad civil. Finalmente, la oposición en el Parlamento terminó por generar una completa crisis de gobernabilidad ya que Sánchez de Lozada no solo no tenía apoyo popular, sino que no podía contar con la clase política tradicional, e incluso el vicepresidente Carlos Mesa se había diferenciado de su gestión. La renuncia del Presidente era inevitable. Cercado, sin apoyo político, acuciado por problemas económicos y sociales, en un ambiente signado de violencia, el vacío de poder se terminó de cristalizar y Sánchez de Lozada dejó el poder y se exilió.

\subsection{El fin de una era. Una nueva crisis de gobernabilidad}

El vicepresidente Carlos Mesa fue reconocido por el Congreso como Presidente de la Nación el 17 de octubre de 2003. Con la idea de ganar parte de la gobernabilidad perdida, pidió apoyo a los congresistas a fin de hacer una democracia más participativa, a partir de la convocatoria a referéndums y enmiendas constitucionales. Principalmente, Mesa buscaba detener las protestas sociales por lo que aceptó discutir algunas de las demandas que provenían de los sectores más movilizados: campesinos e indígenas; de lo contrario, su mandato no hubiera siquiera empezado. En este sentido, es importante destacar la "luna de miel" con la que comenzó su gestión, ya que el $82 \%$ de la población boliviana apoyaba su mandato ${ }^{23}$. Este porcentaje era, lejos, el más alto desde el retorno a la democracia.

El gobierno de Mesa fue definido por muchos como un gobierno de transición histórica, sin participación directa de partidos políticos y con el claro objetivo de preservar la democracia. Uno de los hechos más importantes durante su administración fue la reforma constitucional del año 2004, en la que se reconoce a Bolivia como un Estado multiétnico y pluricultural. En este contexto, con fuertes dificultades de gobernabilidad, el Presidente buscó hacer visibles todas sus acciones y negociar con todos los actores políticos, incluso con el Movimiento al Socialismo (MAS) de Evo Morales ${ }^{24}$.

En ese contexto era necesario mejorar todo lo posible la situación sociopolítica: "Es una coyuntura que ofrece oportunidades para una salida ins- 
titucional a la crisis que implica también riesgos muy importantes debido a la precariedad de la situación política y económica del país", afirmaban Calderón y Gamarra $^{25}$. Por ello, es necesario no desestimar que se haya evitado una intervención cívico-militar, como proponían algunos sectores, al tiempo que se apuntalaban los mecanismos democráticos previstos institucionalmente para llevar adelante una transición ordenada en un escenario que no dejaba de ser caótico.

A pesar del pedido de apoyo de Mesa, los partidos tradicionales se opusieron fervientemente a su gestión, dificultando aún más la gobernabilidad del sistema. Las propuestas de referéndum y Asamblea Constituyente generaron debates en el seno de la clase política pero también en la sociedad civil, que se expresó a través de distintas movilizaciones. En la ya quebrada Bolivia (porque la división entre la zona de la Media luna y el resto del país era un hecho consumado), los departamentos de oriente (la Media luna) se opusieron de cuajo a las propuestas de Mesa porque obstaculizaban la explotación del gas y amenazaban con otorgar más atribuciones a los indígenas ${ }^{26}$. Recién cuando el Presidente consiguió el respaldo de Evo Morales, logró que se aprobara la convocatoria al referéndum. Sin embargo, a pesar de su respaldo a la incorporación de elementos que contribuyeran a una democracia más participativa, Morales no dejó de impulsar las movilizaciones sociales en contra del gobierno, que le sirvieron de base para que su movimiento tuviera alcance nacional ${ }^{27}$.

La intransigente oposición parlamentaria, junto con movilizaciones sociales cada vez más radicales, las presiones del sector empresarial de la zona de la Media luna y de Estados Unidos para erradicar los cultivos de coca, terminaron por cercar a Mesa. La gravedad de la crisis de gobernabilidad que atravesaba su administración quedó clara en la carta de renuncia que presentó al Parlamento, en marzo de año 2005, donde afirmaba que no podía seguir gobernando sitiado, rodeado de amenazas y ultimátums. ${ }^{28}$

Sin embargo, el Congreso rechazó su renuncia, mientras los dirigentes políticos y sindicales se movilizaban para profundizar el clima de protesta social acorralando al Presidente hacia su rendición incondicional. En realidad, lo que perseguían los nuevos actores políticos encolumnados tras la carismática figura de Evo Morales, era dar por tierra con el modelo neoliberal que se había instaurado en los años 90 y reivindicar lo autóctono, la identidad de la Bolivia con base en los pueblos indígenas, capaz de mantener y respetar las diversas culturas

25 CALDERÓN y GAMARRA. Op. cit. p. 15.

26 ARDAYA Salinas, Gloria. La crisis política en Bolivia. Bolivia. Revista Umbrales: Bolivia y el contexto político actual, Nro. 19. 2009, pp.23-46.

27 COTLER, J. Loc. cit.

28 Ibid. 
que la componen, apoyándose en el Estado plurinacional que se reconoció en la reforma constitucional de 2004.

Como consecuencia, un aún más debilitado Presidente presentó nuevamente su renuncia con carácter de indeclinable el 5 de junio de 2005, después de 20 meses de intentar negociar con la oposición parlamentaria y las distintas organizaciones sociales. Para Mesa era imposible seguir gobernando con el apoyo de un pequeño y fragmentado grupo de parlamentarios y con la amenaza de la Central Obrera Boliviana (COB) de conformar un gobierno cívico-militar que lo reemplazara a él ${ }^{29}$.

El sucesor natural de Mesa era el presidente de la Cámara de Senadores, y de no ser posible su nombramiento, le correspondía hacerse del Poder Ejecutivo al titular de la Cámara Baja. Sin embargo, los dirigentes de las organizaciones sociales y políticas más poderosas rechazaron de cuajo esta solución, argumentando que el primero de los candidatos pertenecía a Santa Cruz de la Sierra, y que por lo tanto beneficiaría a la "oligarquía"; y el segundo, era miembro de uno de los partidos tradicionales fuertemente criticados. Como consecuencia, el presidente del Tribunal Supremo, Eduardo Rodríguez, se hizo cargo del gobierno y los legisladores de los partidos tradicionales renunciaron a sus mandatos dos años antes de tiempo. Estas renuncias fueron el resultado de la presión de la opinión pública, que consideraba que estos habían perdido representatividad debido a las abruptas modificaciones en la arena política.

La llegada de Rodríguez a la presidencia representó la derrota de la elite política y de los partidos tradicionales que pretendían que el presidente del Senado, Hormando Vaca Díez, se hiciera cargo del Ejecutivo ${ }^{30}$, y la victoria por parte de los nuevos actores políticos, que llegaron al poder de la mano de Evo Morales en el año 2005.

\section{El impacto de las crisis en la seguridad de la región}

La inestabilidad política generada al interior de Bolivia en el periodo comprendido entre los años 2000 y 2005, en conjunto con la fragilidad intrínseca de su Estado, el debilitamiento y la deslegitimación de las instituciones democráticas, se vio reflejada en la región.

Este reflejo quedó plasmado en la profundización de fenómenos prexistentes, tales como el tráfico de drogas y el contrabando, que afectan indefectiblemente a los países de la región. Más allá de la importancia de mantener a la democracia como un valor central dentro de la Comunidad de Estados America- 
nos, es necesario destacar que el mantenimiento de este régimen se ha convertido en una cuestión de seguridad en sí misma ${ }^{31}$.

Desde que la agenda de seguridad se posicionó al tope de las preocupaciones del subcontinente a principios de este siglo, el tráfico de drogas generado desde Bolivia hacia el resto de la región (sudamericana y norteamericana) se ha consolidado como un problema de suma relevancia. En este sentido es necesario destacar que en la Región Andina se produce el 100\% de la cocaína mundial. Sin duda, el hecho de que Bolivia sea el tercer productor mundial de cocaína (después de Perú y Colombia) adquiere especial relevancia a la hora de entender la importancia que tiene para la seguridad regional.

Como consecuencia, nos parece importante tener en cuenta tres elementos que hacen que Bolivia sea uno de los centros de preocupación mundial en torno al narcotráfico, los dos primeros hacen estrictamente a cuestiones institucionales y de seguridad, mientras que el tercero se refiere a usos y costumbres ancestrales: 1) la fragilidad del Estado y su consecuente dificultad en lo que hace al control del territorio nacional; 2) la porosidad de las fronteras incrementada por la debilidad de las instituciones estatales ${ }^{32}$; y 3) la arraigada tradición en lo referente al consumo de la hoja de coca.

El último punto, muchas veces desestimado, implica que la erradicación del cultivo no es una solución a pesar de que se ha planteado como opción en más de una oportunidad. Es importante entender que una parte de la producción local (que es legal) es utilizada para consumo de gran parte de la población. Ya sea en forma de té medicinal o para mascarla, con el fin de combatir los efectos de la altura o sencillamente como un hábito, gran parte de los bolivianos consumen hoja de coca desde hace cientos de años. En este sentido, aunque el consumo de este tipo no esté directamente relacionado con la producción y el tráfico de cocaína, sí es necesario destacar que es un terreno más fácil de abordar para las organizaciones criminales que han logrado ampliar la superficie cultivada de hoja de coca cuando el consumo ancestral se ha mantenido constante. Es decir, no existe una relación directa entre la producción de hoja de coca para el consumo tradicional y la producción de cocaína; pero sí existe una relación indirecta que tiene que ver con la existencia de las plantaciones y los conoci-

31 Para obtener una visión ampliada de la agenda de seguridad hemisférica se recomienda la lectura de BARTOLOMÉ, Mariano. La Seguridad Internacional en el siglo XXI. Más allá de Wesfalia y Clausewitz. Santiago de Chile, Colección de Investigaciones ANEPE N 14, 2006. A fin de profundizar conocimientos sobre cómo la democracia se convirtió en una cuestión de seguridad, se recomienda la lectura de SAMPÓ, Carolina. Crisis de gobernabilidad, Desafíos a la seguridad regional. Los casos de Bolivia y Ecuador (2000-2005). Alemania. Editorial Académica Española. 2011.

32 SAMPÓ, Carolina. El impacto de los Estados en Proceso de Falla en la Seguridad regional: El caso de Paraguay en el Cono Sur. Tesis de Maestría inédita. Universidad Torcuato Di Tella, 2006. 
mientos a la hora de cuidar la coca, cultivarla y cosecharla. En este sentido, el escenario parece ser mucho más auspicioso para el narcotráfico en Bolivia que en Colombia, donde el consumo de hoja de coca no tiene la misma relevancia.

Siendo la fragilidad del Estado una de las cuestiones más relevantes a tener en cuenta en lo que hace al control territorial y fronterizo de Bolivia, es importante analizar el efecto que tuvieron las crisis de gobernabilidad de los años 2003 y 2005 sobre el tráfico de drogas. En este sentido es indispensable aclarar que consideramos que la crisis institucional comienza realmente en el año 2000, con la llamada Guerra del Agua $^{33}$, pero como a partir de ella no hubo ninguna ruptura institucional, no fue incluida en este trabajo. No obstante, en los indicadores se refleja cómo la lucha contra el narcotráfico se debilitó desde ese año en adelante. Sin duda la puesta en jaque de las instituciones democráticas vigentes, así como la falta de eficiencia por parte del Estado, incrementada en un momento de crisis, tienden a verse reflejadas en una serie de indicadores: en la superficie cultivada ilegalmente con hojas de coca, en el número de personas detenidas por producción ilícita de drogas, en las personas detenidas por tráfico de drogas, en las fábricas y pozas destruidas, y en la cantidad de droga incautada.

Es necesario destacar que la demanda legal de la hoja de coca no se ha visto incrementada en los últimos años. Como consecuencia, el aumento de las superficies cultivadas solo puede atribuirse a la demanda de hoja de coca que es utilizada para convertirla en cocaína. Las zonas en las que se cultiva coca son la región del Chapare, en Cochabamba, y la región de los Yungas, en el departamento de La Paz, donde la mayoría de los cultivos son legales (por lo que el control se hace más difícil).

Entre los años 2000 y 2005, como puede verse en la tabla número 1, se observa un nuevo incremento sostenido de la superficie dedicada al cultivo de coca y de la cantidad de clorhidrato de cocaína producido. En primer lugar llama la atención el crecimiento (36\%) del número de hectáreas cultivadas con hoja de coca entre los años 2000 y 2001 . Este aumento se debe principalmente al retroceso de las políticas de erradicación del cultivo ${ }^{34}$. En segundo término, entre los años 2001 y 2005, el crecimiento de la superficie cultivada se sostuvo, fluctuando entre un $7,4 \%$ y un $8,5 \%$. Si se compara el año 2002 (previo a la primera crisis de gobernabilidad analizada) con el año 2005, puede verse que el cultivo de la hoja de coca creció cerca de un $23 \%$.

33 En el año 2000 se llevó adelante una movilización masiva que exigió al gobierno que cancelara la concesión de la distribución de agua de Cochabamba a una empresa extranjera. Si bien la importancia de este suceso no debe ser desestimada ya que provocó una crisis de gobernabilidad, el hecho de que no se haya conseguido la destitución del Jefe de Estado nos ha llevado a dejarla fuera del análisis. A este episodio se lo denomina "Guerra del Agua".

34 BINLEA. Strategy Report. Department of State's International Narcotics Control, Washington, D.C., 2002. 
Tabla 1

Cultivo estimado de coca y producción de hoja de coca

\begin{tabular}{|c|c|c|c|c|c|c|}
\hline Coca & 2000 & 2001 & 2002 & 2003 & 2004 & 2005 \\
\hline Cultivo Neto (ha) ${ }^{35}$ & 14.600 & $19.900^{36}$ & 21.600 & 23.200 & 24.600 & 26.570 \\
\hline Erradicación (ha) & 7.653 & 9.435 & 11.839 & 10.000 & 8.437 & 6.000 \\
\hline $\begin{array}{c}\text { HCl Potencial } \\
\text { (en toneladas métricas) }\end{array}$ & 43 & 60 & 60 & 60 & 65 & 72 \\
\hline
\end{tabular}

Fuente: Department of State's International Narcotics Control. Strategy Report, Bureau for International Narcotics and Law Enforcement Affairs (BINLEA) 2001-2006.

Si se tienen en cuenta las crisis de gobernabilidad más severas que enfrentó Bolivia, no llama la atención ni el incremento de la superficie cultivada durante los años 2003 y 2005, ni tampoco la caída de la cantidad de hectáreas en las que se erradicó la hoja de coca. Concretamente, en el año de la caída de Sánchez de Lozada la erradicación cayó cerca de un 10\%. Desde ese momento y hasta la renuncia del presidente Mesa, la erradicación continuó cayendo, viéndose reducida casi a la mitad si se comparan los años 2002 y 2005 . Sin ninguna duda, la fragilidad de Estado, en especial en lo que hace al control del territorio nacional y a los movimientos de organizaciones criminales dentro del mismo, se refleja en esos indicadores.

Tabla 2

Número de fábricas de elaboración de cocaína y pozas de maceración de hojas de coca destruidas

\begin{tabular}{|c|c|c|c|c|c|c|}
\hline & 2000 & 2001 & 2002 & 2003 & 2004 & 2005 \\
\hline Fábricas de elaboración de cocaína & 790 & 1.006 & 1.420 & 1.769 & 2.254 & 1.006 \\
\hline $\begin{array}{c}\text { Número de pozas de maceración } \\
\text { destruidas }\end{array}$ & 769 & 1.292 & 1.950 & 2.544 & 3.292 & 1.292 \\
\hline
\end{tabular}

Fuente: Instituto Nacional de Estadística de Bolivia (INEB).

En lo que hace a la cantidad de detenciones provocadas por la producción ilícita de drogas, llama la atención que solo 408 individuos fueron detenidos durante el año 2003 y 547 fueron arrestados en el 2005. Aunque la tendencia fue in crescendo, sorprende la poca cantidad de detenciones, en especial en relación a

35 La relación hoja de coca/clorhidrato de cocaína está estimada en 370 kilogramos de hoja por cada un kilo de $\mathrm{HCl}$ de cocaína en la zona del Chapare y es de 315/1 en Yungas.

36 Hasta el 01/06/2001. 
los kilos de droga producidos y a las fábricas de elaboración de cocaína destruidas ese año. Habiendo destruido en el 2003 alrededor de 1.800 fábricas y 2.550 pozas de maceración de hojas de coca y otras 1.006 fábricas, junto con 1.292 pozas en el 2005, el número de detenciones por producción ilegal de drogas parece muy bajo (Ver tabla 2). Solo la corrupción de funcionarios públicos parece ser la explicación para el bajo número de detenciones, sea porque las organizaciones criminales son avisadas antes de los operativos o porque durante los mismos llegan a "algún arreglo" con las fuerzas involucradas en estas acciones.

Paralelamente, cabe destacar que la mayor parte de las detenciones se realizaron en los departamentos de Cochabamba, donde se concentra gran parte de los cultivos, y de Santa Cruz, que limita con Brasil, por donde saldría gran parte de la droga producida en Bolivia (Ver tabla 3). Esta tendencia se confirma cuando se analizan las detenciones por tráfico ilícito: si en el año 2003, 2.166 personas fueron arrestadas acusadas de este cargo, 862 estaban en Santa Cruz y 522 en Cochabamba. Lo mismo ocurre en el año 2005, de los 2.314 detenidos, 905 se encontraban en Santa Cruz y 524 en Cochabamba.

\section{Tabla 3}

Cantidad incautada de droga, por departamento (en kilogramos)

\begin{tabular}{|c|c|c|c|c|c|c|}
\hline & 2000 & 2001 & 2002 & 2003 & 2004 & 2005 \\
\hline La Paz & 817 & 1.699 & 1.620 & 5.902 & 14.468 & 14.314 \\
\hline Cochabamba & 3.306 & 4.491 & 7.013 & 5.305 & 5.669 & 6.906 \\
\hline Santa Cruz & 4.421 & 2.990 & 2.890 & 7.760 & 3.851 & 22.262 \\
\hline Total & 9.344 & 11.669 & 13.857 & 21.409 & 36.919 & 46.019 \\
\hline
\end{tabular}

Fuente: INEB.

Además de ser productor, Bolivia es un importante país de tránsito, especialmente de cocaína peruana pero también colombiana. La porosidad de sus fronteras y la incapacidad del Estado de ejercer efectivamente su soberanía controlando la totalidad del territorio, en especial en las áreas más remotas, harían que la droga pasara a los cinco países con los que limita. Sin embargo, Brasil parece ser el principal destinatario, mientras que Argentina sería el segundo destino. Para el año 2005 una cantidad creciente de la cocaína que Bolivia producía, así como también de la que transitaba el país, se cree tenía como destino su exportación a Europa, Argentina, Brasil, Chile, Paraguay y México, presuntamente para ser vendida en Estados Unidos ${ }^{37}$.

37 BINLEA. Strategy Report. Department of State's International Narcotics Control, Washington, D.C., 2006. 
Aunque la importancia del tráfico de drogas es, sin duda, incomparable, es necesario no perder de vista otro de los grandes negocios ilícitos que afectan a la seguridad regional: el contrabando. En este sentido, Bolivia es uno de los grandes centros de la región en los que se desarrolla esta actividad. A través de sus fronteras se trafican autos, ropa usada y bombonas de gas, entre otras cosas. Una de las particularidades del país es que no solo recibe cuantiosas sumas de productos provenientes del mercado negro, sino que también "exporta" mercancías a este mismo mercado. A fines de la década del noventa, el contrabando en Bolivia llegó a ser de 1.139 millones de dólares. Sin embargo, con la puesta en vigencia de una nueva Ley de Aduanas (1999), el contrabando cayó primero a 690 millones de dólares en 1999 y en el año 2000 alcanzó solo 290 millones.

En el año 2004 Bolivia perdía alrededor de 160 mil empleos por año, de acuerdo a los datos del Instituto Boliviano de Comercio Exterior (IBCE), y se introducían al país mercancías contrabandeadas por un valor de 700 millones de dólares $^{38}$. De hecho, el IBCE reveló que el contrabando alcanzaba el 10\% del PBI y que el $34 \%$ de las importaciones eran ilegales, por lo que se perdieron más de 250 millones de dólares en tributos no recaudados ${ }^{39}$.

Entre los años 2005 y 2007 Bolivia retrocedió en la lucha contra el contrabando siendo que la importación ilegal se duplicó en relación a la medición del año 2004. El contrabando promedio en ese periodo llegó a ser de 1.200 millones de dólares (el mínimo estimado fue de 1.000 millones y el máximo de 1.300 millones de dólares) ${ }^{40}$.

Contrabando a Bolivia, por país, en millones de dólares (2000-2005)

\begin{tabular}{|c|c|c|c|c|c|c|}
\hline & 2000 & 2001 & 2002 & 2003 & 2004 & 2005 \\
\hline Argentina & 16 & 12,2 & 51,6 & 17,3 & 40,1 & 39,4 \\
\hline Brasil & 114,2 & 80,2 & 64,2 & 50,5 & 83,9 & 102,3 \\
\hline Chile & 22,6 & 13,6 & 23,4 & 25,9 & 35,4 & 57,8 \\
\hline Paraguay & 6,2 & 6,4 & 3 & 5,6 & 4,9 & 4,4 \\
\hline Perú & 7 & 5,2 & 6,1 & 5,5 & 10,6 & 17,7 \\
\hline ZOFRI $^{41}$ & 165,2 & 203,4 & 191,7 & 198 & 179 & 270,4 \\
\hline Total & 331,3 & 321,1 & 340,1 & 302,8 & 353,9 & 492 \\
\hline
\end{tabular}

38 IBCE "Contrabando de Importación en Bolivia supera los 1.000 millones de dólares y afecta 200 mil empleos. 2008 (Fecha de entrada: 15/11/11). Disponible en www.cainco.org.bo/.../18_FORO_CONTRABANDO_CONCLU

39 Ibid.

40 Ibid.

41 Zofri es la zona franca de Iquique, en Chile. 
En la tabla anterior se observan tres puntos importantes muy marcados: primero, el importante incremento del contrabando después de la crisis de gobernabilidad de 2003. En algunos casos duplicó ampliamente los números de las importaciones ilegales entre los años 2000 y 2003, regresando después del 2005 a los niveles marcados por el apogeo del contrabando en la década del 90.

En segundo lugar, el papel que tiene la Zona de Libre Comercio de lquique (ZOFRI) en Chile, en abastecer a Bolivia con productos contrabandeados, ya que el 59\% del contrabando ingresó por allí; Y tercero, que las fronteras terrestres son las más vulnerables, como queda claro en las estadísticas de contrabando desde Brasil (23\%) y, bastante más atrás, Argentina (5\%), aparte de la ZOFRI.

Sobre el último punto es interesante resaltar que los mismos países desde los que se origina el contrabando a Bolivia se han constituido en los últimos años en los principales socios comerciales del país (el 50\% de las importaciones de Bolivia provienen de sus vecinos). Esta situación contribuye al deterioro de las relaciones de intercambio, en especial por el efecto que produce en la economía las ventas de mercaderías ingresadas ilegalmente.

En este contexto, es necesario volver a destacar que tanto la fragilidad del Estado y su incapacidad para ejercer efectivamente la soberanía como los niveles de corrupción, facilitan el tráfico ilícito e ilegal de bienes a través de las fronteras del país. Las áreas grises donde las organizaciones criminales tienen el poder se tornan absolutamente visibles. En este contexto, no existe duda alguna de que el problema más grave que enfrenta Bolivia tiene que ver con la falta de voluntad por parte de la clase política para modificar la situación que data de años previos al retorno a la democracia y parece perpetuarse incluso luego del periodo analizado en este trabajo.

\section{A modo de conclusión}

Tanto la transición a la democracia como la consolidación del régimen en Bolivia estuvieron íntimamente relacionadas con la dinámica que los partidos políticos y la sociedad civil establecieron hasta fines de los años noventa, cuando se corporizó la crisis de representación. En ese contexto, surgieron nuevos partidos políticos (que provenían de diversos movimientos) y se recurrió a la movilización de sectores sociales que se encontraban excluidos de la arena política y demandaban la profundización de la democracia, criticando el sistema político, los mecanismos de representación vigentes y las reformas neoliberales.

Esos nuevos movimientos sociales se hicieron visibles a través de la Política en las Calles, es decir, de la utilización de manifestaciones públicas como instrumentos de presión sobre el sistema político. Este tipo de movilizaciones 
sociales, relacionadas claramente con la falta de confianza en instituciones democráticas claves como los partidos políticos y el Parlamento, hacen que las demandas de la sociedad civil ingresen directamente al sistema político (desde abajo, hacia arriba, es decir, desde el pueblo hacia los gobernantes), posicionándose al tope de la agenda de gobierno, sin pasar por los representantes, considerados meros intermediarios.

No es casual que hayan sido, en última instancia, las movilizaciones sociales masivas las que terminaron por provocar la caída de los presidentes Sánchez de Lozada y Mesa, tras las crisis de gobernabilidad de los años 2003 y 2005, respectivamente.

La debilidad estatal explica muchos de los conflictos presentes en la sociedad boliviana y ha contribuido fuertemente a que se desencadenen las mencionadas crisis de gobernabilidad. En este sentido, la permanente trasgresión de las normas conlleva el debilitamiento extremo de la institucionalidad vigente, complejizando las prácticas sociopolíticas y fomentando la informalidad económica, social y política que caracteriza a Bolivia. Es claro que el Estado está ausente de una importante cantidad de áreas, no posee el monopolio de la fuerza física legítima, ni de la recaudación impositiva, viéndose desplazado por otros poderes. Como consecuencia, su incapacidad redunda en beneficio de otras organizaciones, generalmente criminales como pueden ser los traficantes de drogas o contrabandistas.

Sin duda, la caída de los niveles de gobernabilidad suele traducirse en un aumento de los indicadores de inseguridad regionales, a partir de una lógica simple: el incremento de las dificultades del Estado para mantener el monopolio legítimo de la fuerza física, el control de su territorio y, particularmente, el dominio de los flujos que traspasan sus fronteras, facilita el asentamiento y la proliferación de organizaciones criminales. En el caso de Bolivia en particular, y de Sudamérica en general, la producción y el tráfico de drogas es el problema más acuciante; mientras que en segundo orden de prioridades se encuentra el contrabando. Ambos fenómenos dejan de manifiesto la debilidad de los Estados en lo referente al control del territorio y de las fronteras, así como también demuestran los niveles de corrupción imperantes en distintas agencias gubernamentales.

En este contexto, cabe destacar que durante el periodo analizado, particularmente en los años que llevaron al quiebre institucional, pudo verse (tal como demuestran los números antes expuestos): 1. un incremento en los cultivos ilegales y en la producción de hoja de coca: 2 . una reducción en la cantidad de hectáreas donde se intentó erradicar el cultivo: 3. un bajo número de detenidos considerando la cantidad de pozas y fábricas descubiertas y destruidas. 
Sin duda, estos tres elementos dan cuenta de tres fenómenos distintos pero concatenados.

Por un lado, el incremento de cultivos ilegales de hoja de coca, en un contexto de consumo tradicional sin aumentos que ameriten mayores superficies para la producción y el consumo, pone de manifiesto la demanda generada con fines ilegales. Es decir, si las alteraciones en la demanda no responden al consumo legal y tradicional, es claro que esas plantaciones van a ser utilizadas como insumo para el clorhidrato de cocaína. En este sentido, la imposibilidad por parte del Estado de controlar un territorio de difíciles características geográficas, sumada a su debilidad intrínseca, posibilita el mejor desarrollo de las organizaciones criminales.

El segundo punto, que hace referencia a la reducción de las hectáreas donde se llevó adelante la erradicación, da cuenta de la incapacidad del Estado de poner sus esfuerzos en combatir la expansión de los cultivos, considerando que el gobierno de turno estaba enfrentando una crisis de gobernabilidad de tal envergadura que llevaría al quiebre institucional.

Finalmente, el punto referente al bajo número de detenidos durante operativos de control hace pensar en los altos niveles de corrupción que se manejan en distintas agencias gubernamentales, así como también en los pocos controles que existen en lo que hace a los flujos dentro del territorio y a través de las fronteras.

Con el contrabando pasa algo similar, de acuerdo a los pocos datos fidedignos existentes, los millones de dólares que se movieron en los años de las crisis de gobernabilidad analizadas se vieron incrementados fuertemente a causa de la falta de controles y del beneplácito de algunos funcionarios públicos.

En síntesis, como hemos visto a lo largo del trabajo, tanto la producción de hoja de coca, su transformación en clorhidrato de cocaína y el contrabando se vieron altamente beneficiados por las carencias de los controles estatales. Los gobiernos, sea el dirigido por Sánchez de Lozada o el que llevó adelante Mesa, centraron sus esfuerzos en tratar de manejar las demandas sociales, contener la crisis de representación y pilotar la gobernabilidad tanto en la esfera política como social, desatendiendo cuestiones centrales de la soberanía como el control de los flujos fronterizos y de aquello que ocurre dentro del territorio nacional. Como consecuencia, algunas organizaciones criminales sacaron partido del vacío de poder existente y tomaron control de ciertos sectores territoriales sumamente importantes para la producción y el tráfico de cocaína, aunque también para el contrabando de otros bienes. 


\section{Bibliografía}

ALENDA, Stéphanie. Bolivia: La erosión del pacto democrático. Quito, Ecuador Debate, agosto de 2004. pp. 119-136.

ARDAYA Salinas, Gloria. La crisis política en Bolivia. Bolivia. Revista Umbrales: Bolivia y el contexto político actual, Nro. 19, 2009, pp. 23-46.

BARTOLOMÉ, Mariano. La Seguridad Internacional en el siglo XXI. Más allá de Wesfalia y Clausewitz. Santiago de Chile, Colección de Investigaciones ANEPE, N 14, 2006.

BUREAU FOR INTERNATIONAL NARCOTICS AND LAW ENFORCEMENT AFFAIRS (BINLEA). Strategy Report. Department of State's International Narcotics Control, Washington, D.C., 2002.

BUREAU FOR INTERNATIONAL NARCOTICS AND LAW ENFORCEMENT AFFAIRS (BINLEA). Strategy Report. Department of State's International Narcotics Control, Washington, D.C., 2003.

BUREAU FOR INTERNATIONAL NARCOTICS AND LAW ENFORCEMENT AFFAIRS (BINLEA). Strategy Report. Department of State's International Narcotics Control, Washington, D.C., 2004.

BUREAU FOR INTERNATIONAL NARCOTICS AND LAW ENFORCEMENT AFFAIRS (BINLEA). Strategy Report. Department of State's International Narcotics Control, Washington, D.C., 2005.

BUREAU FOR INTERNATIONAL NARCOTICS AND LAW ENFORCEMENT AFFAIRS (BINLEA). Strategy Report. Department of State's International Narcotics Control, Washington, D.C., 2006.

CALDERÓN, Fernando y GAMARRA, Eduardo. Crisis, inflexión y reforma del sistema de partidos en Bolivia, Revista Colombia Internacional Nro. 58, 2003, pp. 90-123 (Fecha de ingreso: 15/09/10). Disponible en http:// colombiainternacional.uniandes.edu.co/view.php/421/1.php

CAMOU, Antonio. Gobernabilidad y democracia, Cuadernos de divulgación de la cultura democrática Nro. 6, México Instituto Federal Electoral, 2001.

COTLER, Julio. Bolivia-Ecuador-Perú, 2003-2004: ¿Tempestad en los Andes? Real Instituto El Cano de Estudios Internacionales y Estratégicos, Área: América Latina - DT No 51/2005 (Fecha de Consulta: 08/05/11). Disponible en www.realinstitutoelcano.org/documentos/233/233_Cotler.pdf entrado 01/07/10 
CUMBRE EXTRAORDINARIA DE LAS AMÉRICAS (2004). Declaración de Nuevo León. Monterrey, enero de 2004. Disponible en www.summit-americas. org/SpecialSummit/declaration_monterrey-eng.htm entrado 12/08/10

FUNDACIÓN MILENIO (2010). Informe Nacional de Coyuntura Nº 52 Contrabandos en Bolivia. Disponible en http://www.fundacion-milenio.org/documentos/ doc_details/60-informe-nacional-de-coyuntura-no-52-contrabandos-enbolivia.html entrado 20/09/10

GARCíA Montero, Mercedes. Bolivia. En ALCÁNTARA, Manuel y FREIDENBERG, Flavia (coord.) Partidos Políticos de América Latina. Países Andinos. México, D.F., Fondo de Cultura Económica e Instituto Federal Electoral, 2003, pp. 33-145.

HERRERO, Francisco. Sistema de Partidos y desarrollo: El caso de Bolivia. En GUERRA García, Gustavo y SAMPLE, Kristen (coeditores). La política y la pobreza en los países andinos. Lima, International Institute for Democracy and Electoral Assistance y Asociación Civil Transparencia, 2007.

INCHUTA Nina, Carlos E. La relación de las instituciones políticas, la forma multitud y los movimientos sociales en Bolivia. Tinkazos, vol. 11 Nro. 25, La Paz, noviembre 2008 (Fecha de ingreso: 15/08/10). Disponible en http://www.scielo.org.bo/scielo.php?script=sci_arttext\&pid=S1990$74512008000200008 \&$ Ing $=e s \& n r m=i s s \& t$ Ing $=e s$

INSTITUTO BOLIVIANO DE COMERCIO EXTERIOR (IBCE). "Contrabando de Importación en Bolivia supera los 1.000 millones de dólares y afecta 200 mil empleos. 2008 (Fecha de entrada: 15/11/11). Disponible en www. cainco.org.bo/.../18_FORO_CONTRABANDO_CONCLU

LASERNA, Roberto. Gobernabilidad Democrática y Reforma Política en Bolivia. Brasil, Fundación Milenio, Documento Preparado para la Fundación Konrad Adenauer, 2003.

MORENO Morales, Daniel. La marcha nuestra de cada día: normalización de la protesta en Bolivia. En: MORENO Morales, Daniel et al. Cultura Política en tiempos de cambio. Institucionalidad, conflicto y región en Bolivia. Cochabamba. Observatorio de Cultura Política/Ciudadanía/LAPOP, 2009, pp. 71-90.

O’DONNELL, Guillermo. El Estado Burocrático Autoritario. Triunfos, derrotas y crisis. Bs. As. Editorial de Belgrano, 1996.

ORGANIZACIÓN DE LOS ESTADOS AMERICANOS (OEA) "Declaración de Managua para la Promoción de la Democracia y el Desarrollo" (AG/DEC. 4 (XXIII-O/93), 1993. 
ORGANIZACIÓN DE LOS ESTADOS AMERICANOS (OEA). Carta Democrática Interamericana. Aprobada en la primera sesión plenaria de la Asamblea General Extraordinaria, Lima, 11 de septiembre de 2001.

ORGANIZACIÓN DE LOS ESTADOS AMERICANOS (OEA). Declaración de Santiago sobre Democracia y Confianza Ciudadana: un nuevo compromiso de gobernabilidad para las Américas. AG/DEC. 31 (XXXIII-O/03), 10 de junio de 2003.

ORGANIZACIÓN DE LOS ESTADOS AMERICANOS (OEA). Declaración sobre Seguridad en las Américas, OEA/Ser.K/XXXVIII, 28 de octubre de 2003.

ORGANIZACIÓN DE LOS ESTADOS AMERICANOS (OEA). Programa de Gobernabilidad Democrática en las Américas. AG/RES. 1960 (XXXIII-O/03), 10 de junio de 2003.

OSSIO Bustillos, Teresa y PRATS Catala, Joan. Crisis de gobernabilidad como oportunidad democrática: el caso de Bolivia. En Congreso Internacional del CLAD sobre la Reforma del Estado y de la Administración Pública (IX Madrid, España, 2-5 Nov. 2004).

PNUD (compilador). Escenarios políticos en América Latina. Conceptos, métodos y observatorio regional, Bs. As., Siglo XXI Editores, 2008.

ROJAS Ortuste, Gonzalo. Bolivia ante el cambio: ¿Reforma pactada o revolucionarismoetnicista? Bolivia, Revista Umbrales Nro. 19: Bolivia y el contexto político actual, 2009, pp. 47-78.

SAMPÓ, Carolina. El impacto de los Estados en Proceso de Falla en la Seguridad regional: El caso de Paraguay en el Cono Sur. Tesis de Maestría, inédita. Universidad Torcuato Di Tella, 2006.

SAMPÓ, Carolina. Crisis de gobernabilidad, Desafíos a la seguridad regional. Los casos de Bolivia y Ecuador (2000-2005). Alemania. Editorial Académica Española, 2011.

SUÁREZ, Hugo. Seis claves para interpretar la crisis boliviana. Memoria № 198 , México, agosto de 2005 (Fecha de Ingreso 20/03/11). Disponible en http:// catedras.fsoc.uba.ar/toer/articulos/txt-suarez.htm

TOMASSINI, Luciano. Gobernabilidad y políticas públicas en América Latina. En CARRILLO Flores, Fernando (ed.). Democracia en déficit. Gobernabilidad y desarrollo en América Latina, Washington, D.C., BID, 2001, p. 71. 
VARGAS R., Humberto y CORDOVA E., Eduardo. Bolivia: un país de reconfiguraciones por una cultura de pactos políticos y de conflictos. En SEOANE, José. Movimientos sociales y conflictos en América Latina, Buenos Aires, CLACSO, 2003, p. 85.

WALDMANN, Peter. El Estado Anómico. Derecho, seguridad pública y vida cotidiana en América Latina. Caracas, Nueva Sociedad, 2003.

WITKER Barra, Iván. Bolivia 2003 en la prensa chilena. Percepciones de la crisis en la prensa chilena y su impacto en la seguridad subregional y relaciones bilaterales. Santiago de Chile, Colección de Investigaciones ANEPE N ${ }^{\circ}$ 11, 2005. 